\title{
ABOUT ONE PROBLEM ON EXTREMAL DECOMPOSITION
}

\begin{abstract}
In the paper, we consider an open problem of finding the maximum of product of inner radii of mutually non-overlapping domains with respect to the points of the unit circle on a certain positive degree $\gamma$ of the inner radius of the domain with respect to the origin, moreover, the domain containing origin does not intersect with other domains.
\end{abstract}

Key words: inner radius of the domain, mutually non-overlapping domains, the Green function, quadratic differential, the Dubinin problem

\section{Mathematical Subject Classification: 30C75}

1. Preliminaries. Let $\mathbb{N}, \mathbb{C}$ be the sets of natural and complex numbers, respectively, $\overline{\mathbb{C}}=\mathbb{C} \bigcup\{\infty\}$ be its one-point compactification, $\mathbb{R}^{+}=(0, \infty)$. Let $B$ be a domain in $\overline{\mathbb{C}}$. Let

$$
g_{B}(z, a)=h_{B, a}+\log \frac{1}{|z-a|}+o(1)
$$

be the Green function of the domain $B$ with respect to a point $a \in B$. Quantity $r(B, a):=\exp \left(h_{B, a}\right)$ is called an inner radius of the domain $B \subset \overline{\mathbb{C}}$ with respect to a point $a \in B$ (see, for example, [6], [9], [11], [15]).

Let $n \in \mathbb{N}, n \geqslant 2$. A set of points $A_{n}:=\left\{a_{k} \in \mathbb{C}: k=\overline{1, n}\right\}$ is called an $n$-radial system if $\left|a_{k}\right| \in \mathbb{R}^{+}$and $0=\arg a_{1}<\ldots<\arg a_{n}<2 \pi$. Denote the numbers $\alpha_{k}, k=\overline{1, n}$ as follows: $\alpha_{1}:=\frac{1}{\pi}\left(\arg a_{2}-\arg a_{1}\right)$, $\alpha_{2}:=\frac{1}{\pi}\left(\arg a_{3}-\arg a_{2}\right), \ldots, \alpha_{n}:=\frac{1}{\pi}\left(2 \pi-\arg a_{n}\right)$. Obviously, $\sum_{k=1}^{n} \alpha_{k}=2$. Let $\alpha_{0}=\max _{k} \alpha_{k}$.

Consider the following problem, which was formulated in [9], [10] in the list of unsolved problems.

(C) Petrozavodsk State University, 2021 
The Dubinin Problem. Prove that the maximum of the functional

$$
I_{n}(\gamma)=r^{\gamma}\left(B_{0}, a_{0}\right) \prod_{k=1}^{n} r\left(B_{k}, a_{k}\right)
$$

where $B_{0}, B_{1}, B_{2}, \ldots, B_{n},(n \geqslant 2)$ are pairwise non-overlapping domains in $\overline{\mathbb{C}}, a_{0}=0,\left|a_{k}\right|=1, k=\overline{1, n}, a_{k} \in B_{k}, k=\overline{0, n}$ and $\gamma \leqslant n$, is attained at a configuration of domains $B_{k}$ and points $a_{k}$ possessing rotational $n$ symmetry.

In the paper [9] the above-formulated problem was solved for $\gamma=1$ and all values of the natural parameter $n \geqslant 2$. Namely, it was shown that the following inequality holds:

$$
r\left(B_{0}, 0\right) \prod_{k=1}^{n} r\left(B_{k}, a_{k}\right) \leqslant r\left(D_{0}, 0\right) \prod_{k=1}^{n} r\left(D_{k}, d_{k}\right),
$$

where $d_{k}, D_{k}, k=\overline{0, n}$, are the poles and circular domains of the quadratic differential

$$
Q(w) d w^{2}=-\frac{\left(n^{2}-1\right) w^{n}+1}{w^{2}\left(w^{n}-1\right)^{2}} d w^{2} .
$$

In the work [14], Kovalev got the solution for systems of points for which the following inequalities hold:

$$
\left|a_{k}\right|=1, \quad 0<\alpha_{k} \leqslant 2 / \sqrt{\gamma}, \quad k=\overline{1, n}, \quad n \geqslant 5 .
$$

In the work [3], it was shown that the result by Kovalev is also true for $n=4$. In the monograph [2] the problem was solved for an arbitrary $\gamma>1$ but starting from some previously unknown number $n$. The next step was to study this problem at the restrictions $1<\gamma \leqslant n^{\delta}$, where $0<\delta<1$ (see, for example [7], [8], [18-20]).

In the paper [5], the authors obtained its complete solution for $n=2$. Note that the result of [5] is a consequence of the well-known theorem of Kolbina [13].

Let

$$
I_{n}^{0}(\gamma):=r^{\gamma}\left(D_{0}, 0\right) \prod_{k=1}^{n} r\left(D_{k}, d_{k}\right),
$$

where $d_{k}, D_{k}, k=\overline{0, n}, d_{0}=0$, be, respectively, the poles and circular domains of the quadratic differential

$$
Q(w) d w^{2}=-\frac{\left(n^{2}-\gamma\right) w^{n}+\gamma}{w^{2}\left(w^{n}-1\right)^{2}} d w^{2}
$$


As has been shown in Theorem 5.2.3 [2], the quantity $I_{n}^{0}(\gamma)$ takes the form

$$
I_{n}^{0}(\gamma)=\left(\frac{4}{n}\right)^{n} \frac{\left(\frac{4 \gamma}{n^{2}}\right)^{\frac{\gamma}{n}}}{\left(1-\frac{\gamma}{n^{2}}\right)^{n+\frac{\gamma}{n}}}\left(\frac{1-\frac{\sqrt{\gamma}}{n}}{1+\frac{\sqrt{\gamma}}{n}}\right)^{2 \sqrt{\gamma}} .
$$

Theorem 1. [5] Let $\gamma \in(1,2]$. Then, for any different points $a_{1}$ and $a_{2}$ of the unit circle and any pairwise non-overlapping domains $B_{0}, B_{1}, B_{2}$, $a_{0}=0 \in B_{0} \subset \overline{\mathbb{C}}, a_{1} \in B_{1} \subset \overline{\mathbb{C}}, a_{2} \in B_{2} \subset \overline{\mathbb{C}}$, the following inequality holds:

$$
r^{\gamma}\left(B_{0}, 0\right) r\left(B_{1}, a_{1}\right) r\left(B_{2}, a_{2}\right) \leqslant I_{2}^{0}(\gamma)\left(\frac{1}{2}\left|a_{1}-a_{2}\right|\right)^{2-\gamma} .
$$

The sign of equality is attained when the points $a_{0}, a_{1}, a_{2}$ and the domains $B_{0}, B_{1}, B_{2}$ are, respectively, the poles and circular domains of the quadratic differential

$$
Q(w) d w^{2}=-\frac{(4-\gamma) w^{2}+\gamma}{w^{2}\left(w^{2}-1\right)^{2}} d w^{2}
$$

Besides, in the paper [5] the solution for $n \geqslant 3$ and $\gamma \in(1, \sqrt{n}$ ] is obtained using an upper estimate for the functional $I_{n}(\gamma)$ [4].

Theorem 2. [5] Let $n \in \mathbb{N}, n \geqslant 3, \gamma \in(1, \sqrt{n}]$. Then, for any system of different points $A_{n}=\left\{a_{k}\right\}_{k=1}^{n}$ of a unit circle and for any collection of mutually non-overlapping domains $B_{0}, B_{k}, a_{0}=0 \in B_{0} \subset \overline{\mathbb{C}}, a_{k} \in B_{k} \subset \overline{\mathbb{C}}$, $k=\overline{1, n}$, the following inequality holds:

$$
r^{\gamma}\left(B_{0}, 0\right) \prod_{k=1}^{n} r\left(B_{k}, a_{k}\right) \leqslant r^{\gamma}\left(D_{0}, 0\right) \prod_{k=1}^{n} r\left(D_{k}, d_{k}\right),
$$

where $d_{k}, D_{k}, k=\overline{0, n}, d_{0}=0$, are, respectively, the poles and circular domains of the quadratic differential (1).

Also, in [5] the following upper estimate for $I_{n}(\gamma)$ is proved for $n \geqslant 3$ and $\gamma \in(1, n]$ :

Theorem 3. [5] Let $n \in \mathbb{N}, n \geqslant 3, \gamma \in(1, n]$. Then, for any system of different points $A_{n}=\left\{a_{k}\right\}_{k=1}^{n}$ of a unit circle and for any collection of mutually non-overlapping domains $B_{0}, B_{k}, a_{0}=0 \in B_{0} \subset \overline{\mathbb{C}}, a_{k} \in B_{k} \subset \overline{\mathbb{C}}$, $k=\overline{1, n}$, the inequality

$$
r^{\gamma}\left(B_{0}, 0\right) \prod_{k=1}^{n} r\left(B_{k}, a_{k}\right) \leqslant\left(\sin \frac{\pi}{n}\right)^{n-\gamma}\left(I_{2}^{0}\left(\frac{2 \gamma}{n}\right)\right)^{\frac{n}{2}}
$$


holds.

In deriving our results, the following estimates specified below are required:

Lemma 1. [1] Let $n \in \mathbb{N}, n \geqslant 2, \gamma>0$. Let $\left\{B_{0}, B_{1}, B_{2}, \ldots, B_{n}\right\}$ be a system of mutually non-overlapping simply connected domains, such that $0 \in B_{0} \subset \overline{\mathbb{C}}, a_{k} \in B_{k} \subset \overline{\mathbb{C}},\left|a_{k}\right|=1, k=\overline{1, n}$, and $r^{\gamma}\left(B_{0}, 0\right) \prod_{k=1}^{n} r\left(B_{k}, a_{k}\right)>I_{n}^{0}(\gamma)$. Then the following inequality holds:

$$
r\left(B_{0}, 0\right) \leqslant n^{-\frac{n}{2(n-\gamma)}} I_{n}^{0}(\gamma)^{-\frac{1}{n-\gamma}} .
$$

Lemma 2. [4] Let $n \in \mathbb{N}, n \geqslant 2, \gamma \in(0, n]$. Then for any system of different fixed points $A_{n}=\left\{a_{k}\right\}_{k=1}^{n} \subset \mathbb{C} \backslash\{0\}$, such that $\prod_{k=1}^{n}\left|a_{k}\right| \leqslant 1$, and for any collection of mutually non-overlapping domains $\left\{B_{0}, B_{1}, B_{2}, \ldots, B_{n}\right\}$, such that $a_{0}=0, a_{k} \in B_{k} \subset \overline{\mathbb{C}}, k=\overline{0, n}$, the following inequality holds:

$$
I_{n}(\gamma) \leqslant n^{-\frac{\gamma}{2}}\left(I_{n}(0)\right)^{1-\frac{\gamma}{n}} .
$$

According to the condition of the problem $a_{0}=0,\left|a_{k}\right|=1, k=\overline{1, n}$, further, we assume without loss of generality that $0=\arg a_{1}<\arg a_{2}<$ $\ldots<\arg a_{n}<2 \pi$. Since in [14] the problem was solved under conditions $0<\alpha_{k} \leqslant 2 / \sqrt{\gamma}, k=\overline{1, n}, n \geqslant 5$, then, for a given $n$, it is sufficient to consider only configurations of domains $B_{k}$ and points $a_{k}$ for which $\alpha_{0}>\frac{2}{\sqrt{\gamma}}$. The results of this paper are addendum to the theorem of the work [14].

2. Main results. In this work, we prove the following propositions.

Theorem 4. Let $n \in \mathbb{N}, n \geqslant 24$ and $1<\gamma \leqslant n^{\frac{2}{3}-\frac{2}{3} \frac{\ln (\ln (n))}{\ln (n)}}$. Then the following inequality holds:

$$
r^{\gamma}\left(B_{0}, a_{0}\right) \prod_{k=1}^{n} r\left(B_{k}, a_{k}\right) \leqslant\left(\frac{4}{n}\right)^{n} \frac{\left(\frac{4 \gamma}{n^{2}}\right)^{\frac{\gamma}{n}}}{\left(1-\frac{\gamma}{n^{2}}\right)^{n+\frac{\gamma}{n}}}\left(\frac{1-\frac{\sqrt{\gamma}}{n}}{1+\frac{\sqrt{\gamma}}{n}}\right)^{2 \sqrt{\gamma}}
$$

where $B_{k}, k=\overline{0, n}$, are mutually non-overlapping simply connected domains in $\overline{\mathbb{C}}, a_{0}=0,\left|a_{k}\right|=1, k=\overline{1, n}$, and the equality is attained, in particular, for points $a_{k}$ and domains $B_{k}$ that are, respectively, the poles and circular domains of the quadratic differential (1). 
Proof. Using Lemma 1 and the result of the paper [14], consider the case $\alpha_{0}>\frac{2}{\sqrt{\gamma}}$ and $r\left(B_{0}, 0\right) \leqslant n^{-\frac{n}{2(n-\gamma)}} I_{n}^{0}(\gamma)^{-\frac{1}{n-\gamma}}$. Prove that under these conditions

$$
\frac{r^{\gamma}\left(B_{0}, a_{0}\right) \prod_{k=1}^{n} r\left(B_{k}, a_{k}\right)}{\left(\frac{4}{n}\right)^{n} \frac{\left(\frac{4 \gamma}{n^{2}}\right)^{\frac{\gamma}{n}}}{\left(1-\frac{\gamma}{n^{2}}\right)^{n+\frac{\gamma}{n}}}\left(\frac{1-\frac{\sqrt{\gamma}}{n}}{1+\frac{\sqrt{\gamma}}{n}}\right)^{2 \sqrt{\gamma}}}<1 .
$$

Further, from Lemma $1 r^{\gamma}\left(B_{0}, a_{0}\right) \leqslant n^{-\frac{n \gamma}{2(n-\gamma)}} I_{n}^{0}(\gamma)^{-\frac{\gamma}{n-\gamma}}$. Then, using Theorem 5.2.3 [2], the following estimates hold:

$$
\begin{aligned}
& \prod_{k=1}^{n} r\left(B_{k}, a_{k}\right) \leqslant 2^{n} \prod_{k=1}^{n} \alpha_{k} \leqslant 2^{n} \alpha_{0}\left(\frac{2-\alpha_{0}}{n-1}\right)^{n-1}< \\
&<\frac{4^{n}}{(n-1)^{n-1} \sqrt{\gamma}}\left(1-\frac{1}{\sqrt{\gamma}}\right)^{n-1} .
\end{aligned}
$$

And, thus, we obtain the inequality

$$
\frac{I_{n}(\gamma)}{I_{n}^{0}(\gamma)} \leqslant \frac{\frac{4^{n}}{(n-1)^{n-1} \sqrt{\gamma}}\left(1-\frac{1}{\sqrt{\gamma}}\right)^{n-1}}{n^{\frac{n \gamma}{2(n-\gamma)}} I_{n}^{0}(\gamma)^{\frac{n}{n-\gamma}}}:=G_{n}(\gamma)
$$

Combining the previous inequality and inequality (2), we get

$$
\begin{aligned}
& G_{n}(\gamma)=n^{\frac{n \gamma+2 n+2 \gamma}{2(n-\gamma)}}\left(\frac{n}{n-1}\right)^{n-1}\left(1-\frac{1}{\sqrt{\gamma}}\right)^{n-1}\left(1-\frac{\gamma}{n^{2}}\right)^{\frac{n^{2}+\gamma}{n-\gamma}} \times \\
& \times\left(\frac{1}{4 \sqrt{\gamma}}\right)^{\frac{n+\gamma}{n-\gamma}}\left(\frac{1+\frac{\sqrt{\gamma}}{n}}{1-\frac{\sqrt{\gamma}}{n}}\right)^{\frac{2 n \sqrt{\gamma}}{n-\gamma}} .
\end{aligned}
$$

Note that in order to prove Theorem 4 , we need to show that $G_{n}(\gamma)<1$ for given $n$ and $\gamma$.

Evaluate the expression $G_{n}(\gamma)$ under the conditions of the theorem. It is not difficult to show that $\left(\frac{n}{n-1}\right)^{n-1}<e$ and $\left(1-\frac{\gamma}{n^{2}}\right)^{\frac{n^{2}+\gamma}{n-\gamma}}<1$. Also, the assessment $\left(\frac{1+\frac{\sqrt{\gamma}}{n}}{1-\frac{\sqrt{\gamma}}{n}}\right)^{\frac{2 n \sqrt{\gamma}}{n-\gamma}}\left(\frac{1}{4 \sqrt{\gamma}}\right)^{\frac{n+\gamma}{n-\gamma}}<0,06<\frac{1}{e}$ is correct. Accordingly,

$$
G_{n}(\gamma)<n^{\frac{n \gamma+2 n+2 \gamma}{2(n-\gamma)}}\left(1-\frac{1}{\sqrt{\gamma}}\right)^{n-1}
$$


Evaluate the expression $n^{\frac{n \gamma+2 n+2 \gamma}{2(n-\gamma)}}\left(1-\frac{1}{\sqrt{\gamma}}\right)^{n-1}$. We get the following transformations:

$$
\begin{gathered}
n^{\frac{n \gamma+2 n+2 \gamma}{2(n-\gamma)}}\left(1-\frac{1}{\sqrt{\gamma}}\right)^{n-1}=n^{\frac{n \gamma+2 n+2 \gamma}{2(n-\gamma)}}\left(\left(1-\frac{1}{\sqrt{\gamma}}\right)^{\sqrt{\gamma}}\right)^{\frac{n-1}{\sqrt{\gamma}}}< \\
<\left(n\left(\frac{1}{e}\right)^{\frac{2 n^{2}-2 n-2 n \gamma+2 \gamma}{n \gamma \sqrt{\gamma}+2 n \sqrt{\gamma}+2 \gamma \sqrt{\gamma}}}\right)^{\frac{n \gamma+2 n+2 \gamma}{2(n-\gamma)}}=\left(n\left(\frac{1}{e}\right)^{\frac{n}{\gamma^{\frac{3}{2}}} \frac{2-\frac{2}{n}-\frac{2 \gamma}{n}+\frac{2 \gamma}{n}}{1+\frac{2}{\gamma}+\frac{2}{n}}}\right)^{\frac{n \gamma+2 n+2 \gamma}{2(n-\gamma)}} .
\end{gathered}
$$

Since in the paper [18] the problem is solved for $n \geqslant 12$ and $1<\gamma \leqslant n^{0,45}$, it is enough to consider only $\gamma>n^{0,45}$.

For $n \geqslant 24$ and $n^{0,45}<\gamma<n^{\frac{2}{3}-\frac{2}{3} \frac{\ln (\ln (n))}{\ln (n)}}$, the inequality

$$
\frac{2-\frac{2}{n}-\frac{2 \gamma}{n}+\frac{2 \gamma}{n^{2}}}{1+\frac{2}{\gamma}+\frac{2}{n}}>1
$$

is satisfied. So, we have

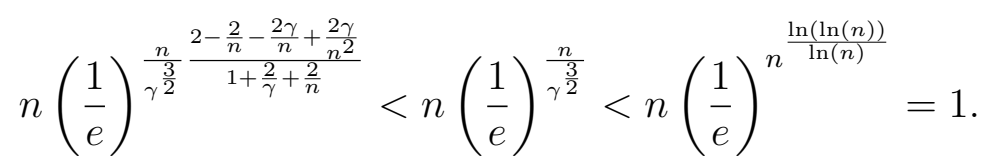

Thus, from inequality (4) we obtain that for $n$ and $\gamma$ given in Theorem 4 $G_{n}(\gamma)<1$, which means that

$$
r^{\gamma}\left(B_{0}, a_{0}\right) \prod_{k=1}^{n} r\left(B_{k}, a_{k}\right) \leqslant I_{n}^{0}(\gamma)
$$

Theorem 4 is proved.

Theorem 5. Let $n \in \mathbb{N}, n \geqslant 12$ and $1<\gamma \leqslant n^{\frac{2}{3}-\frac{2}{3} \frac{\ln (2 \ln (n))}{\ln (n)}}$. Then the result of the Theorem 4 remains valid without the condition of simply connected domains $B_{k}, k=\overline{0, n}$.

Proof. Taking into account the paper [14], consider the case $\alpha_{0}>\frac{2}{\sqrt{\gamma}}$. Prove that under this condition

$$
\frac{r^{\gamma}\left(B_{0}, a_{0}\right) \prod_{k=1}^{n} r\left(B_{k}, a_{k}\right)}{I_{n}^{0}(\gamma)}<1 .
$$


Then, using Theorem 5.2.3 [2], we obtain

$$
\prod_{k=1}^{n} r\left(B_{k}, a_{k}\right)<\frac{4^{n}}{(n-1)^{n-1} \sqrt{\gamma}}\left(1-\frac{1}{\sqrt{\gamma}}\right)^{n-1} .
$$

Thus, using inequality (3) and the previous inequality,

$$
\begin{aligned}
r^{\gamma}\left(B_{0}, a_{0}\right) & \prod_{k=1}^{n} r\left(B_{k}, a_{k}\right)< \\
& <n^{-\frac{\gamma}{2}} 4^{n-\gamma}\left(\frac{1}{n-1}\right)^{\frac{n^{2}-n-n \gamma+\gamma}{n}}\left(\frac{1}{\gamma}\right)^{\frac{n-\gamma}{2 n}}\left(1-\frac{1}{\sqrt{\gamma}}\right)^{\frac{n^{2}-n-n \gamma+\gamma}{n}} .
\end{aligned}
$$

So, we have the inequality

$$
\begin{aligned}
\frac{I_{n}(\gamma)}{I_{n}^{0}(\gamma)} \leqslant & 4^{-\gamma-\frac{\gamma}{n}} n^{n-\frac{\gamma}{2}+\frac{2 \gamma}{n}}\left(\frac{1}{\gamma}\right)^{\frac{n+\gamma}{2 n}}\left(\frac{1}{n-1}\right)^{\frac{n^{2}-n-n \gamma+\gamma}{n}} \times \\
& \times\left(1-\frac{1}{\sqrt{\gamma}}\right)^{\frac{n^{2}-n-n \gamma+\gamma}{n}}\left(1-\frac{\gamma}{n^{2}}\right)^{n+\frac{\gamma}{n}}\left(\frac{1+\frac{\sqrt{\gamma}}{n}}{1-\frac{\sqrt{\gamma}}{n}}\right)^{2 \sqrt{\gamma}}:=P_{n}(\gamma) .
\end{aligned}
$$

It is easy to see that

$$
n^{n-\frac{\gamma}{2}+\frac{2 \gamma}{n}}\left(\frac{1}{n-1}\right)^{\frac{n^{2}-n-n \gamma+\gamma}{n}}=n^{\gamma+1+\frac{\gamma}{n}}\left(\frac{n}{n-1}\right)^{n-1-\gamma+\frac{\gamma}{n}} .
$$

Taking into account the previous equality, we get the following expression:

$$
\begin{aligned}
& P_{n}(\gamma)=4^{-\gamma-\frac{\gamma}{n}}\left(\frac{1}{\gamma}\right)^{\frac{n+\gamma}{2 n}} n^{\gamma+1+\frac{\gamma}{n}}\left(1-\frac{1}{\sqrt{\gamma}}\right)^{\frac{n^{2}-n-n \gamma+\gamma}{n}} \times \\
& \times\left(\frac{n}{n-1}\right)^{n-1-\gamma+\frac{\gamma}{n}}\left(1-\frac{\gamma}{n^{2}}\right)^{n+\frac{\gamma}{n}}\left(\frac{1+\frac{\sqrt{\gamma}}{n}}{1-\frac{\sqrt{\gamma}}{n}}\right)^{2 \sqrt{\gamma}} .
\end{aligned}
$$

Since in the paper [18] the problem is solved for $n \geqslant 12$ and $1<\gamma \leqslant n^{0,45}$, it is enough to consider only $\gamma>n^{0,45}$. Evaluate the expression $P_{n}(\gamma)$ under the conditions of the theorem. Using estimates

$$
\left(\frac{n}{n-1}\right)^{n-1-\gamma+\frac{\gamma}{n}}<e, \quad\left(1-\frac{\gamma}{n^{2}}\right)^{n+\frac{\gamma}{n}}<1,
$$




$$
\left(\frac{1+\frac{\sqrt{\gamma}}{n}}{1-\frac{\sqrt{\gamma}}{n}}\right)^{2 \sqrt{\gamma}} 4^{-\gamma-\frac{\gamma}{n}}\left(\frac{1}{\gamma}\right)^{\frac{n+\gamma}{2 n}}<0,06<\frac{1}{e}
$$

we get

$$
P_{n}(\gamma)<n^{\gamma+1+\frac{\gamma}{n}}\left(1-\frac{1}{\sqrt{\gamma}}\right)^{\frac{n^{2}-n-n \gamma+\gamma}{n}}
$$

Evaluate the expression $n^{\gamma+1+\frac{\gamma}{n}}\left(1-\frac{1}{\sqrt{\gamma}}\right)^{\frac{n^{2}-n-n \gamma+\gamma}{n}}$ under the conditions of the theorem. The following transformations are also correct:

$$
\begin{gathered}
n^{\gamma+1+\frac{\gamma}{n}}\left(1-\frac{1}{\sqrt{\gamma}}\right)^{\frac{n^{2}-n-n \gamma+\gamma}{n}}=n^{\gamma+1+\frac{\gamma}{n}}\left(\left(1-\frac{1}{\sqrt{\gamma}}\right)^{\sqrt{\gamma}}\right)^{\frac{n^{2}-n-n \gamma+\gamma}{\sqrt{n \gamma}}}< \\
<\left(n\left(\frac{1}{e}\right)^{\frac{n^{2}-n-n \gamma+\gamma}{n \gamma \sqrt{\gamma}+n \sqrt{\gamma}+\gamma \sqrt{\gamma}}}\right)^{\gamma+1+\frac{\gamma}{n}}=\left(n\left(\frac{1}{e}\right)^{\frac{n}{\gamma^{\frac{3}{2}}} \frac{1-\frac{1}{n}-\frac{\gamma}{n}+\frac{\gamma}{n^{2}}}{1+\frac{1}{\gamma}+\frac{1}{n}}}\right)^{\gamma+1+\frac{\gamma}{n}} .
\end{gathered}
$$

For $n \geqslant 12$ and $n^{0,45}<\gamma<n^{\frac{2}{3}-\frac{2}{3} \frac{\ln (2 \ln (n))}{\ln (n)}}$, the inequality

$$
\frac{1-\frac{1}{n}-\frac{\gamma}{n}+\frac{\gamma}{n^{2}}}{1+\frac{1}{\gamma}+\frac{1}{n}}>0,5
$$

holds. Consequently,

$$
\begin{aligned}
& n\left(\frac{1}{e}\right)^{\frac{n}{\gamma^{\frac{3}{2}}} \frac{1-\frac{1}{n}-\frac{\gamma}{n}+\frac{\gamma}{n^{2}}}{1+\frac{1}{\gamma}+\frac{1}{n}}}<n\left(\frac{1}{e}\right)^{\frac{n}{2 \gamma^{\frac{3}{2}}}}<
\end{aligned}
$$

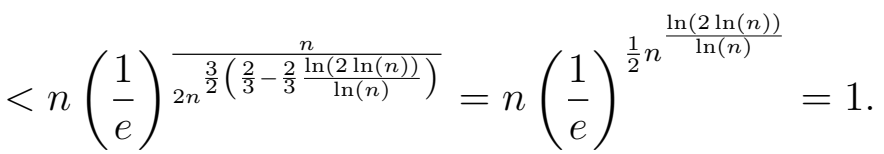

Thus, from inequality (5) we obtain that for $n$ and $\gamma$ given in Theorem 5 $P_{n}(\gamma)<1$, that is

$$
r^{\gamma}\left(B_{0}, a_{0}\right) \prod_{k=1}^{n} r\left(B_{k}, a_{k}\right) \leqslant I_{n}^{0}(\gamma) .
$$

Theorem 5 is proved.

Acknowledgements. The authors thank the anonymous referees whose comments helped to improve and clarify this manuscript. 


\section{References}

[1] Bakhtin A. K. Estimates of inner radii for pairwise non-overlapping domains. Zb. prats of the Inst. of Math. of NASU. 2017, vol. 14, no. 1, pp. $25-33$.

[2] Bakhtin A. K., Bakhtina G. P., Zelinskii Yu. B. Topological-algebraic structures and geometric methods in complex analysis. $\mathrm{Zb}$. prats of the Inst. of Math. of NASU, 2008. (in Russian)

DOI: https://doi.org/10.13140/RG.2.1.1660.6242

[3] Bakhtin A. K., Denega I. V. Addendum to a theorem on extremal decomposition of the complex plane. Bulletin de la sociétédes sciences et des lettres de Lódź, Recherches sur les déformations. 2012, vol. 62, no. 2, pp. 83-92.

[4] Bakhtin A. K., Denega I. V. Inequalities for the inner radii of nonoverlapping domains. Ukr. Math. J. 2019, vol. 71, pp. 1138-1145.

DOI: https://doi.org/10.1007/s11253-019-01703-x

[5] Bakhtin A. K., Denega I. V. Extremal decomposition of the complex plane with free poles. J. Math. Sci. 2020, vol. 246, no. 1, pp. 1-17.

DOI: https://doi.org/10.1007/s10958-020-04718-z

[6] Goluzin G. M. Geometric theory of functions of a complex variable. Amer. Math. Soc. Providence, R.I., 1969.

[7] Denega I.V., Zabolotnii Ya.V. Estimates of products of inner radii of nonoverlapping domains in the complex plane. Complex Variables and Elliptic Equations. 2017, vol. 62, no. 11., pp. 1611-1618.

DOI: https://doi.org/10.1080/17476933.2016.1265952

[8] Denega I., Zabolotnii Ya. Problem on extremal decomposition of the complex plane. An. St. Univ. Ovidius Constanta. 2019, vol. 27, no. 1., pp. $61-77$.

DOI: https://doi.org/10.2478/auom-2019-0004

[9] Dubinin V. N. Symmetrization method in geometric function theory of complex variables. Russian Math. Surveys. 1994, vol. 1, pp. 1-79.

DOI: http://dx.doi.org/10.1070/RM1994v049n01ABEH002002

[10] Dubinin V. N. Condenser capacities and symmetrization in geometric function theory. Birkhäuser/Springer, Basel, 2014.

DOI: https://doi.org/10.1007/978-3-0348-0843-9

[11] Hayman V. Multivalent functions. Cambridge University Press, Cambridge, 1958.

[12] Jenkins J. Univalent functions and conformal mapping. Moscow:Publishing House of Foreign Literature, 256, 1962. (in Russian)

DOI: https://doi.org/10.1007/978-3-642-88563-1 
[13] Kolbina L. I. Conformal mapping of a unit circle onto nonoverlapping domains. Vestn. Lenin. Univ., 1955, vol. 5, pp. 37-43. (in Russian)

[14] Kovalev L. V. On the problem of extremal decomposition with free poles on a circle. Dal'nevost. Mat. Zh., 1996, no. 2, pp. 96-98. (in Russian)

[15] Lavrent'ev M. A. On the theory of conformal mappings. Tr. Sci. Inst An USSR, 1934, vol. 5, pp. 159-245. (in Russian)

[16] Lebedev N. A. The area principle in the theory of univalent functions. Moscow, Science, 1975 (in Russian).

[17] Tamrazov P. M. Extremal conformal mappings and poles of quadratic differentials. Izv. Akad. Nauk SSSR Ser. Mat. 1968, vol. 32, no. 5., pp. $1033-1043$.

[18] Zabolotnii Ya. V. Some application of the method of separating transformation in one problem on nonoverlapping domains. Dopov. Nac. akad. nauk Ukr. 2011, no. 9, pp. 11-14.

[19] Zabolotnii Ya., Denega I. On Conformal Radii of Non-Overlapping Simply Connected Domains. International Journal of Advanced Research in Mathematics. 2018, vol. 11, no. 11., pp. 1-7.

DOI: https://doi.org/10.18052/www.scipress.com/IJARM.11.1

[20] Zabolotnii Ya., Dvorak I. Some evaluation of maximum of the product of conformal radii for pairwaise nonoverlapping domains. Lobachevskii Journal of Mathematics. 2017, vol. 38, no. 6, pp. 554-559.

DOI: https://doi.org/10.1134/S1995080217030271

Received June 10, 2021.

In revised form, July 26, 2021.

Accepted August 08, 2021.

Published online August 23, 2021.

Institute of Mathematics of the National Academy of Sciences of Ukraine 3, Tereschenkivska st., Kyiv-4 01024, Ukraine

Yaroslav Zabolotnii

yaroslavzabolotnii@gmail.com,

Iryna Denega

iradenega@gmail.com 